\title{
Vollzug des Umweltschutzgesetzes in den Bereichen Luftreinhaltung und Bodenschutz - Anliegen an die Adresse der angewandten Forschung
}

\section{Einleitung}

Thema dieses Beitrages sind die mit dem Umweltschutzgesetz (USG) verbundenen Vollzugsaufgaben in den Teilbereichen Luftreinhaltung und gütemäßiger (qualitativer) Bodenschutz. Dabei soll aufgezeigt werden, wo die «beamteten Umweltschützer» in Bund, Kantonen und Gemeinden auf Sachverstand angewiesen sind, der nicht zuletzt von den Geographen erwartet wird.

\section{Was heißt «Vollzug»?}

\section{Die drei Bedeutungen des Begriffs}

«Vollzug» ist ein schillernder Begriff. Er kann bedeuten:

- Konkretisierung einer Vorschrift durch den Erlaß von Ausführungsrecht

- Konkretisierung einer Vorschrift durch Anordnungen im Einzelfall

- Durchsetzung des Rechts («Zähmung der Widerspenstigen»)

\section{Erlaß von Ausführungsverordnungen}

Gesetzesbestimmungen sind notwendigerweise stark abstrakt formuliert. Im Extremfall zeigt der Gesetzgeber mit seiner Formulierung im Grunde nur an, daß ein bestimmtes Problem besteht. Wie die Lösung genau aussehen soll, wie stark z. B. die Verwaltung den Bürgern und Bürgerinnen «auf die Zehen treten» darf, steht zuweilen nicht präzis im Gesetz.

Das USG ist voll von solchen Programmnormen, Generalklauseln, Delegationsnormen und unbestimmten Rechtsbegriffen. Beispiele:

"Schädliche und lästige Einwirkungen», "wirtschaftliche Tragbarkeit», «Unverhältnismäßigkeit einer Sanierung im Einzelfall», «Bodenfruchtbarkeit», «Störung des Wohlbefindens», «Der Bundesrat erläßt Vorschriften über umweltgefährdende Stoffe».

Es versteht sich, daß mit solchen Vorschriften allein die praktischen Fragen nicht gelöst sind. Sowohl die betroffenen Privaten als auch die Behörden müssen genauer wissen, was gemeint ist. In diesem Sinn meint Vollzug, die abstrakten Aussagen im Gesetz durch Ausführungsverordnungen «auszudeutschen». So nahm der Bundesrat - um die vorstehenden Beispiele wieder aufzunehmen - in der Zwischenzeit folgende Konkretisierungen vor:

- Präzise Immissionsgrenzwerte geben an, wann Einwirkungen «schädlich oder lästig» sind oder das «Wohlbefinden erheblich stören»;

- präzise Emissionsgrenzwerte zeigen an, welcher Aufwand bei der Emissionsbegrenzung an der Quelle als «wirtschaftlich tragbar» gilt;

- eine ausführliche Definition umschreibt den Begriff der «Bodenfruchtbarkeit» 1 ;

- eine Verordnung mit 76 Artikeln und 19 Anhängen enthält ausführliche «Vorschriften über Stoffe»2; sie formuliert zahlreiche Gebote und Verbote, führt Bewilligungspflichten und Anmeldeverfahren ein und weist die Behörden im Detail an, was und wie zu kontrollieren ist.

Der «Vollzug» in diesem Sinn - d. h. der Erlaß von konkretisierendem Recht auf unterer Stufe - ist primär Aufgabe des Bundesrates.

\section{Fallweise Anordnungen}

Selbst die detaillierteste Verordnung ist noch immer «abstrakt», d. h. sie preßt die komplexe Wirklichkeit in (möglichst wenige) Sätze, die jeweils nach folgendem Schema aufgebaut sind: «Wenn sich der Sachverhalt A ereignet, dann knüpft daran die Rechtsfolge B.»

Beispiel: Wer eine Feuerung betreiben will, darf nur Öl, Gas oder Brennholz verwenden.

Was aber - so fragt (um im zitierten Beispiel zu bleiben) bestimmt ein besorgter Bürger (nennen wir ihn Herr Tanner) die Behörde - ist «Brennholz»? Herr Tanner besitzt nämlich einen mit Kunststoff furnierten Tisch aus Tannenholz, der ihm nicht mehr gefällt, weshalb er ihn in seinem Ofen verfeuern möchte. Ob er das dürfe?

"Nein», wird ihm die Behörde antworten (oder sollte es jedenfalls): «Unter Brennholz sei unbeschichtetes (genauer: nicht mit Kunststoffen beschichtetes) Holz zu verstehen. Herr Tanner dürfe

André Schrade, Fürsprecher, Koordinationsstelle für Umweltschutz Basel-Stadt, Martinsgasse 12, 4001 Basel 
also seinen Tisch nicht in seinem Heizofen verbrennen, sondern müsse ihn der Abfuhr übergeben.»

Die Behörde hat hier die abstrakte Norm auf einen konkreten Einzelfall angewandt - was übrigens nie ohne Interpretation abgeht. Ihre Anordnung ist nunmehr sehr präzis: sie ist individuell (d. h. richtet sich konkret an Herrn Tanner und nur an ihn) und sie ist konkret (d. h. sie gilt für den erwähnten Tisch und nur für diesen Tisch). In der Rechtssprache heiBen solche individuell-konkreten Anordnungen «Verfügungen».

Solche Verfügungen zu erlassen, d. h. maßgeschneiderte Lösungen für den einzelnen Fall zu definieren, gehört ebenfalls zum Vollzug. Diese Aufgabe fällt nach dem USG - mit wenigen Ausnahmen den Kantonen zu, die ihrerseits die Gemeinden damit betrauen können.

Für die Kantone bedeutet dies, daß sie eine entsprechende Behördenorganisation aufstellen und die Kompetenzen verteilen müssen. Sie sind darin weitgehend frei (sog. Organisationshoheit), was zu äuBerst originellen Lösungen führt. Es bieten sich im wesentlichen drei Modelle für die Behördenorganisation an (wobei ich hier bewußt karikiere):

- Das «Modell Tessin»: Als (bisher) einziger Kanton hat der Tessin für den Umweltschutz ein eigenes Departement eingerichtet. Es faßt alle mit dem Umweltschutz beauftragten Fachstellen unter einem administrativen Hut zusammen. $\mathrm{Da}$ Umweltschutz eine stark querschnittsbezogene Materie darstellt, müßte freilich tendenziell die ganze Verwaltung in diesem Departement zusammengefaßt werden ...

- Das «Modell Chaos»: Die neuen Bundesaufgaben werden fallweise derjenigen Fachstelle «angehängt», die sich bereits «irgendwie» mit dem fraglichen Sachgebiet beschäftigt. So könnte die Bekämpfung des Straßenverkehrslärms dem StraBenbauinspektorat (bei Autobahnen: dem Autobahnamt), der Schießlärm dem Militärdepartement, der Gewerbelärm dem Amt für Industrie, Gewerbe und Arbeit, der Baulärm dem Baudepartement usw. angegliedert werden. Natürlich braucht nun jede Fachstelle je einen Akustiker, einen Meßwagen, eine Fachbibliothek usw. Jede Stelle arbeitet isoliert vor sich hin, was zu sehr bunten Vollzugsergebnissen führen wird.

- Das Modell «Koordination»: Es beruht auf dem Modell «Chaos», doch entsenden die Vorsteher aller betroffenen Amtsstellen (nicht nur der spezifisch mit Umweltschutzaufgaben betrauten Behörden, sondern z. B. auch der Bauämter und des Amtes für den Straßenverkehr) je einen Vertreter in ein Gremium, für das sich der Name «Umweltschutzkommission» anbietet. Der Vorsitzende dieser Kommission verfügt über einen eigenen kleinen Stab und hat die Aufgabe, die Vollzugsaufgaben zu koordinieren. Folgerichtig wird die- ser Stab als «Koordinationsstelle für Umweltschutz» bezeichnet.

Der Trend dürfte in die Richtung des Modells «Koordination» gehen (z. B. Zürich, Bern, BaselStadt, Luzern, Aargau). Sieht man vom Pionierkanton Tessin ab, so steht meines Wissens zurzeit einzig im Kanton Basel-Landschaft ein Projekt für die Schaffung eines Umweltschutzdepartementes konkret zur Diskussion.

\section{Durchsetzung des Rechts (Zähmung der Widerspenstigen)}

Schließlich meint Vollzug, dafür zu sorgen, daß die Betroffenen ihren Rechtspflichten auch wirklich nachleben. Das bedingt Kontrollen - was im Umweltschutz vorab heißt: Messungen -, sei es durch die Behörde selber oder in ihrem Auftrag durch Private.

Werden Verstöße festgestellt, so müssen Sanktionen angedroht und wenn nötig durchgesetzt werden. $\mathrm{Zu}$ den Sanktionen gehören nicht nur die strafrechtlichen Mittel, sondern auch die Mittel des sog. Verwaltungszwanges, etwa das Instrument der Ersatzvornahme: Die vom Recht verlangte Maßnahme wird auf Kosten des Säumigen von der Behörde selber durchgeführt. (Natürlich wird der Säumige oft auch die Rechnung nicht bezahlen, weshalb die Behörde dann zum sog. Vollstreckungszwang - hier: zur Betreibung - schreiten muss.)

Die Erfahrung lehrt, daß der Einsatz von Verwaltungs- und Vollstreckungszwang erstens unerfreulich und zweitens für die Behörde ausgesprochen arbeitsintensiv ist. Konsequente Renitenz bringt den Vollzug in arge Schwierigkeiten. Mag der Begriff der «Akzeptanz» staatlicher Maßnahmen auch häufig mißbraucht werden: Im Kern benennt er eine durchaus reelle Problemlage des Vollzugs.

Auch dieser Aspekt des Vollzugs («Vollzug im engeren Sinne») ist im Umweltschutz primär Sache der Kantone oder - in ihrem Auftrag - der Gemeinden.

\section{Vollzug im Bereich Luftreinhaltung}

\section{Was ist zu vollziehen?}

Zusammengefaßt geht es im Vollzug (i.w.S.) darum, das im USG verankerte zweistufige Konzept der Luftreinhaltung zu verwirklichen: Vorsorge zum einen, Abwehr übermäßiger Immissionen zum andern.

In einer ersten Stufe sollen die Emissionen an Luftschadstoffen durch Maßnahmen bei der Quelle (also bei den Anlagen) ganz unabhängig von der Höhe der Gesamtbelastung so weit begrenzt werden, als dies technisch und betrieblich möglich und wirtschaftlich tragbar ist. 
«Unabhängig von der bestehenden Umweltbelastung» heißt, daß die Emissionen auch bei den Anlagen in jenen Gebieten zu begrenzen sind, die (noch) keine übermäßige Gesamtbelastung (Immissionen) aufweisen. In diesem Sinn verwirklicht die erste Stufe das sog. Vorsorgeprinizp.

Mit Maßnahmen der ersten Stufe allein gelingt es freilich nicht immer, übermäßige Immissionsbelastungen zu verhindern bzw. genügend abzubauen. Oft ist die Vorbelastung bereits viel zu hoch, oder der Erfolg der ersten Maßnahmestufe wird durch die ständige Zunahme der Quellen (Motorfahrzeuge!) gleich wieder «aufgefressen».

Deshalb sieht das USG eine zweite Maßnahmenstufe vor: Die Emissionen bei der Quelle müssen schärfer begrenzt werden, wenn feststeht oder zu erwarten ist, daß die Einwirkungen schädlich oder lästig sind bzw. würden. Konkret heißt dies, daß die wirtschaftliche Tragbarkeit keine Schranke mehr für die Schärfe der Emissionsbegrenzungen bei der Quelle darstellt.

\section{Folgerungen für das Ausführungsrecht}

Das Gesetz verwendet im Teilbereich Luftreinhaltung zwei idealtypische «unbestimmte Rechtsbegriffe»: Den Begriff der «wirtschaftlichen Tragbarkeit» und den Ausdruck «schädliche oder lästige Einwirkungen» (i. S. von Immissionen). Aufgabe des Bundesrates ist es nun, durch Verordnung die nötige Konkretisierung zu leisten. Mit der Luftreinhalte-Verordnung (LRV) hat er diese Aufgabe - für den Moment wenigstens - erfüllt. («Für den Moment» deshalb, weil der Stand der Technik fortschreitet und die Wissenschaft laufend neue Erkenntnisse über die Schadwirkung von Luftfremdstoffen liefert.)

\section{Folgerungen für den Erlaß von Verfügungen}

Maßgeschneiderte Verfügungen sind vor allem gegenüber den Inhabern von Altanlagen nötig. Denn die LRV nennt zwar präzise Emissions- und Immissionsgrenzwerte, doch kann die nötige Sanierung nicht von heute auf morgen erfolgen. Es müssen deshalb in jedem Einzelfall gerechte Fristen angeordnet werden. Dafür sind - wie bereits erwähnt wurde - die Kantone (und Gemeinden) zuständig.

\section{Folgerungen für die Durchsetzung (Vollzug im engeren Sinn)}

Die LRV zu vollziehen, heißt:

- Messen, Rechnen, Schätzen (Emissionen und Immissionen);

- Vergleichen des Ergebnisses mit den rechtlichen Vorgaben und

- den widerspenstigen Anlageninhabern notfalls «Beine machen».
Diese Aufgabe obliegt zur Hauptsache den Kantonen (und Gemeinden) und ist ebenso aufwendig wie fachlich anspruchsvoll. Vor allem muß ermittelt werden, ob Maßnahmen der ersten (vorsorglichen) Stufe ausreichen oder ob verschärfte Emissionsbegrenzungen verlangt werden dürfen/müssen. Wenn ja: Welche Quellen müssen härter angefaßt werden? Welchen Beitrag muß die einzelne Quelle an die insgesamt nötige Reduktion der Immissionsbelastung leisten? Die Behörden müssen hier planmäßig vorgehen, d. h. - in der Terminologie der Luftreinhalte-Verordnung - einen Maßnahmenplan aufstellen.

\section{Folgerungen für die Forschung}

Das zentrale Problem lautet: Wann ist eine Immission schädlich oder lästig. Davon hängt schließlich $\mathrm{ab}$, ob die erste oder die zweite, verschärfte Maßnahmenstufe zum Zuge kommt.

Maßgeblich sind - wird der Jurist sagen - die Immissionsgrenzwerte im Anhang zur Luftreinhalte-Verordnung. Natürlich! Den Naturwissenschafter interessiert aber, wie diese Werte zustande kamen. Das Bundesamt für Umweltschutz hat darüber in seinem «Bericht Immissionsgrenzwerte» ${ }^{3}$ ausführlich Rechenschaft abgelegt. Der Bericht ist ehrlich. Er unterschlägt $\mathrm{z}$. B. nicht, daß die Festlegung einer «Unbedenklichkeitsschwelle» (no-effect-level) für bestimmte Schadstoffe - vorab für die kanzerogenen - zurzeit schwierig, wenn nicht überhaupt unmöglich ist. Und er legt offen, daß uns die Wissenschaft (Toxikologie, Biologie, Chemie usw.) häufig noch ratlos läßt, wenn wir nach der Schadwirkung von Luftfremdstoffen fragen, die in Kombination auftreten.

Bedeutende Wissenslücken bestehen auch zur Beantwortung der Frage, welche Anlagen in welchem $\mathrm{Ma} ß$ für eine bestimmte Immissionsbelastung verantwortlich sind. Das gilt namentlich beim Ozon, das bekanntlich nicht tel quel emittiert wird, sondern sich aus bestimmten Vorläufersubstanzen $\left(\mathrm{NO}_{x}\right.$ und $\left.\mathrm{HC}\right)$ unter dem Einfluß komplexer Abläufe in der Chemie der Atmosphäre bildet. Wir wissen einigermaßen, wie dies im makronomischen Bereich abläuft, müssen aber noch genauere Kenntnisse der klein- und großräumigen Transmissionsbedingungen gewinnen. Denn die Behörde steht unter dem Zwang, die Notwendigkeit ihrer Anordnungen beweisen zu können, wenn diese «beschwerdefest» sein sollen. Ich erachte diese Kausalitätsprobleme als die größte Krux des Vollzugs, namentlich bei der Aufstellung der Maßnahmenpläne für die Sanierung der Altanlagen.

Bei der Festlegung der Immissionsgrenzwerte liegt ein weiteres Problem darin, daß die Luft häufig nur das «Zwischenlager» für die Luftfremdstoffe ist. Sie gelangen durch Auswaschung und Trockendeposition schließlich in das «Endlager» Boden. Damit ist 
die Brücke zum zweiten Thema des Referates - dem Bodenschutz - geschlagen.

\section{Vollzug im Bereich Bodenschutz}

\section{Was ist zu vollziehen?}

Dem Gesetzgeber ist nicht entgangen, daß es nicht genügt, den Menschen vor der direkten Vergiftung durch Abgase und Chemikalien zu schützen. Wir würden bekanntlich auch dann aussterben, wenn sich die Schadstoffe im Boden so anreicherten, daß er seine Fruchtbarkeit verlöre. Deshalb weist das USG die Behörden an, die Luftreinhalte- und die Chemikalien-Politik so zu betreiben, daß sie auch der Zielsetzung des qualitativen Bodenschutzes der Erhaltung der Bodenfruchtbarkeit - dient.

\section{Folgen für das Ausführungsrecht}

Es muß Klarheit darüber herrschen, welche Schadstoffgehalte man dem Boden ohne Nachteile für die Bodenfruchtbarkeit zumuten darf. Das ist Sache des Bundesrates. Er hat diesen Auftrag in der Bodenschutz-Verordnung (VSBo) für den Moment erfüllt: Die Verordnung nennt in einem Anhang präzise Richtwerte für zehn Schwermetalle und für Fluor.

Diese Werte haben erstens auf die Immissionsgrenzwerte nach der Luftreinhalte-Verordnung und zweitens auf die Vorschriften in der Stoff-Verordnung (z. B. auf die Qualitätsanforderungen für Dünger) durchgeschlagen. Schon vorher wurde - noch im Rahmen der Gewässerschutzgesetzgebung - eine besondere Verordnung über die Qualität des Klärschlammes erlassen.

Das Problem liegt primär bei den nicht-abbaubaren (persistenten) Stoffen, vorab bei den Schwermetallen. Weil sie sich im Boden laufend anreichern, kann der Grenzwert für den Schwermetallgehalt im Dünger (inkl. Klärschlamm) längerfristig im Grunde nur bei Null liegen: alles andere führt auf die Dauer zu einer Überbelastung. Nimmt man z. B. die heutigen Werte in der Klärschlamm-VO, so läßt sich absehen, daß - bei gleichbleibenden Emissionen - der Boden in etwa 100 Jahren «ausgedient» hätte. Diese beängstigende Erkenntnis wird uns wohl zwingen, Klärschlamm zunehmend zu verbrennen und die Schlacke zu deponieren. Der Bodenschutz trägt mithin die Züge eines fatalen Wettlaufes gegen die Uhr.

\section{Folgerungen für den Erlaß von Verfügungen}

Der Bodenschutz ist insofern ein Spezialfall, als man die Richtwerte nicht direkt «vollziehen» kann:
Sie sind ja gedacht als ein Hilfsmittel für die richtige Dimensionierung der Maßnahmen in anderen Bereichen der Umweltschutz-Politik, namentlich bei der Luftreinhaltung und beim Umgang mit Chemikalien.

Bodenschutz zu treiben, heißt folglich für den Vollzug, Emissionsbegrenzungen für eine bestimmte Anlage anzuordnen oder eine konkrete Verhaltensvorschrift für den Umgang mit Chemikalien in einem bestimmten Gebiet zu erlassen (etwa ein Verbot, Kunstdünger oder Klärschlamm auszubringen). Von der Natur der Sache her kommen dafür nur die vor Ort zuständigen Kantone in Frage.

\section{Folgerungen für die Durchsetzung (Vollzug i.e.S.)}

Hier gibt es nichts Neues zu vermerken: Durchgesetzt wird der Bodenschutz mit dem Mittel des Luftreinhalte- und Chemikalienrechts. In beiden Fällen sind die Kantone (evtl. Gemeinden) angesprochen.

\section{Ein Wort zu NABO}

Das «A und O» des Bodenschutzes ist - wie bei der Luftreinhaltung - die Kenntnis der aktuellen Belastung, die nur durch Messungen, Berechnungen und Schätzungen gewonnen werden kann.

Der Bundesrat hat erkannt, daß er den Aufwand für die nötigen Erhebungen nicht ganz auf die Kantone abschieben darf: Schließlich braucht er die Ergebnisse auch, um seine eigenen Vollzugsaufgaben zu erfüllen (Erlaß der Ausführungsvorschriften über die Luftreinhaltung [Immissionsgrenzwerte!] und der Stoff-Vorschriften [z.B. Begrenzung des Schwermetallgehaltes im Dünger]).

Der Bundesrat hat deshalb die Eidg. Forschungsanstalt für Agrikulturchemie und Umwelthygiene (FAC) in Liebefeld bei Bern beauftragt, ein nationales Meßnetz zur Bodenüberwachung (NABO) aufzuziehen. Bei den Meßpunkten im bewaldeten Boden steht der FAC zudem die Eidg. Anstalt für das forstliche Versuchswesen (EAfV) in Birmensdorf beratend zur Seite, um die Koordination mit dem Programm zur Waldschadens-Beobachtung (SANA-SILVA) sicherzustellen.

Geplant sind insgesamt $100 \mathrm{Meßplätze} \mathrm{an} \mathrm{möglichst}$ repräsentativen Standorten in der ganzen Schweiz. Es gilt, einerseits landwirtschaftlich genutzte Böden im Flachland und in den Alpen sowie Waldböden zu untersuchen. Bis heute sind 38 Meßorte eingerichtet.

NABO wird auch nach seinem Vollausbau ein relativ weitmaschiges Referenznetz (ähnlich dem Programm NABEL zur Luftüberwachung) bleiben. Um einen konkreten Eingriff gegenüber einem Privaten rechtfertigen zu können, werden die Kantone das Netz wesentlich verfeinern müssen. Selbstver- 
ständlich werden sie die Priorität auf Messungen in Gebieten legen, in denen eine relativ hohe Vorbelastung erwartet wird, d. h. auf die Agglomerationen und die Umgebung von bedeutenden Punktquellen (etwa KVA, Buntmetallwerke). Wir rechnen damit, daß in den nächsten Jahren rund 500 Meßstellen aufgezogen werden.

\section{Anliegen an die Forschung}

Der Festlegung der Richtwerte für die zulässigen totalen Gehalte an Schadstoffen in der Verordnung über Schadstoffe im Boden (VSBo) gingen umfangreiche Literaturstudien voraus. Das Bundesamt für Umweltschutz stützte sich schließlich vorab auf die Werte, bei deren Erreichung nach Publikationen des deutschen Forschers KLOKE ${ }^{4}$ eine Beeinträchtigung der Bodenfruchtbarkeit vermutet werden muß. Sie wurden sicherheitshalber halbiert (sog. «halber KLOKE-Wert»).

Die Werte für den Gehalt an leichtlöslichen Schadstoffen wurden von der FAC zum Teil selber erarbeitet. Sie beruhen auf Gefäßversuchen mit Pflanzen und bodenbiologischen Untersuchungen. Auch hier wurde ein Sicherheitsfaktor 2 eingebaut.
An einer kürzlich durchgeführten Fachtagung zum NABO hat Herr HÄNl von der FAC auf einige Wissenslücken hingewiesen. Ich zitiere: «Trotz des Sicherheitsfaktors von zwei bleibt doch die Frage offen, ob man mit den Richtwerten der VSBo für die wichtigsten Kulturpflanzen tatsächlich auf der sicheren Seite liegt. Weiter fehlen neben den Untersuchungen über Wechselwirkungen (Synergismus, Antagonismus) auch die Einzelrichtwerte für die löslichen Gehalte der Schwermetalle Chrom, Kobalt, Molybdän, Quecksilber und Thallium.» Ein weites Feld für die Forschung also!

\section{Anmerkungen}

1 Verordnung über Schadstoffe im Boden (VSBo) vom 9. Juni 1986, Art. 2

2 Verordnung über umweltgefährdende Stoffe (Stoffverordnung, StoV) vom 9. Juni 1986

3 Bundesamt für Umweltschutz, 1986: Immissionsgrenzwerte für Luftschadstoffe. Schriftenreihe Umweltschutz Nr. 52, Bern, 77 S.

4 Kloke, A., 1982: Erläuterungen zur Klärschlammverordnung. Landwirtschaftliche Forschung, Sonderhefte, Nr. 39 , Kongreßband 1982 